Meta

Journal des traducteurs

Translators' Journal

\title{
Défini, indéfini
}

\section{André Clas}

Volume 12, numéro 2, juin 1967

URI : https://id.erudit.org/iderudit/002297ar

DOI : https://doi.org/10.7202/002297ar

Aller au sommaire du numéro

Éditeur(s)

Les Presses de l'Université de Montréal

ISSN

0026-0452 (imprimé)

1492-1421 (numérique)

Découvrir la revue

Citer cet article

Clas, A. (1967). Défini, indéfini. Meta, 12(2), 39-44.

https://doi.org/10.7202/002297ar

Ce document est protégé par la loi sur le droit d'auteur. L’utilisation des services d'Érudit (y compris la reproduction) est assujettie à sa politique d'utilisation que vous pouvez consulter en ligne.

https://apropos.erudit.org/fr/usagers/politique-dutilisation/
Cet article est diffusé et préservé par Érudit.

Érudit est un consortium interuniversitaire sans but lucratif composé de l’Université de Montréal, l'Université Laval et l'Université du Québec à Montréal. Il a pour mission la promotion et la valorisation de la recherche. https://www.erudit.org/fr/ 


\section{DÉFINI. INDÉFINI}

Bien sûr, on lit. Dans les intervalles on lit Don Quichotte, Les Mémoires d'Outre-tombe ...

- Pourquoi dites-vous on?

- Pour ne pas dire tout le temps je. 1
À ce qu'on m'a dit, il s'agirait d'une épidémie de ferraille.

- Qui \& on*?

- J'ai reçu des instructions secrètes.2

Ces citations montrent deux des différentes utilisations du même pronom que la grammaire traditionnelle qualifie d'indéfini. Et nous ne pouvons nous empêcher de penser à J. Marouzeau lorsqu'il écrit: «Est-il rien de plus fréquent, de plus utile, et on peut dire de plus français que l'indéfini on? Nous faisons du pronom un usage abondant et varié. ${ }^{3}$ Certes, "on " est d'un emploi fort commode, mais ce pronom qui se greffe sur le système normal des pronoms personnels ne risque-t-il pas de faire sombrer cette belle logique des sujets différenciés? N'y a-t-il pas là superfétation et illogisme ? Or, ... mais ceci est un autre problème. Nous sommes donc en droit de nous demander comment les locuteurs et les allocutaires s'y retrouvent et comment les traducteurs réussissent à interpréter les différentes valeurs de cette même unité de traduction.

Pour étudier les options à l'intérieur des «cadres permissifs de la langue ${ }^{4}$, nous avons essayé de collectionner le plus grand nombre d'exemples possible en dépouillant systématiquement différents textes de langues de départ, français, anglais, allemand, et les langues d'arrivée respectives, et nous avons étudié comment l'indéfini «on », ce «cadre vide ${ }^{5}$, selon l'expression de Bröndal, acquérait un sens, comment l'incertitude était levée, quelle possibilité en jeu se réalisait.

Dès que le locuteur, pour une raison quelconque, se refuse à mettre en cause quelqu'un ou ne peut mettre en cause quelqu'un, il a recours à « on». Le pronom

1. «Ça crève, ça crève 》, Express, no 755, 6-12 décembre 1965, p. 123.

2. «Affaire Ben Barka. Les $X$ de Santeny 》, Express, n' 758,27 décembre $1965-2$ janvier 1966, p. 15.

3. J. Marouzeau, Aspects du français, Paris, Masson et Cle, 1950, p. 202.

4. B. Pottier, Systématique des éléments de relation, Paris, Klincksieck, 1953, p. 24.

5. V. Bröndal, les Parties du discours, Copenhague, Munksgaard, 1948, p. 116. 
indéfini, par sa modestie, sa prudence, évite au locuteur la nécessité d'une prise de conscience absolue, d'un jugement catégorique. Cette grande souplesse explique peut-être son succès.

On ne l'a pas poussée.6

She wasn't pushed.

Man hat sie nicht gestossen.
C'est ce qu'on m'a dit.7

That's what I was told.

Man hat mir das erzählt.

Dans ces deux exemples, «on » est un universel: il est impossible de lui assigner une valeur spécifique. C'est un pronom de généralisation qui englobe tout un ensemble de personnes, c'est «nous », c'est « ils». Il y a là toute une imprécision affective où le lecteur et le narrateur sont associés et deviennent en quelque sorte complices. L'anonymat des sujets transforme ces affirmations presque en vérités absolues.

La LA anglaise doit nécessairement rendre l'indéfini par la voix passive, car la langue anglaise ne connaît pas d'équivalent et toute l'attention du lecteur est concentrée sur l'expression verbale. Peut-on dire que, dans cette transposition, on = voix passive, la LA enregistre une certaine perte ? Nous ne le croyons pas, car, au fond, la présence en français d'un pronom sujet n'est que formelle. Psychologiquement, les phrases françaises sont impersonnelles au même titre que le passif anglais.

L'allemand possède le même outil que le français: à «on », il répond par «man ». Nous pouvons donc affirmer ici l'identité des valeurs des deux pronoms. La même grisaille, le même recul devant l'explicite se retrouvent dans les deux langues. Nous devons à présent examiner ce qui se passe lorsque l'anglais devient LD. Logiquement, nous devrions pouvoir affirmer la réciprocité de nos démonstrations:

His murderer was never caught. ${ }^{8}$

On n'a jamais arrêté l'assassin.

The trigger was pressed.9

Der Mörderer wurde nie ermittelt.

On pressa la détente.

Der Revolver wurde abgedrückt.

L'anglais a envisagé ici la réalité objectivement en concentrant l'attention du lecteur sur l'expression verbale, sur le procès. Le mode de pensée de la langue anglaise semble ici encore exiger que le sujet, pour être évoqué de façon concrète, figure en position privilégiée, donc à la première place. L'objet et le sujet se condensent pour rejeter vers la fin l'élément verbal plus lourd, donnant ainsi un rythme plus léger et plus agréable à la phrase.

L'allemand reste fidèle à la $L D$ et communique, lui aussi, la réalité par la voix passive. Nous pouvons donc, dès à présent, schématiser nos observations de la façon suivante:

6. G. Simenon, les Vacances de Maigret, Paris, Les Presses de la cité, 1951, p, 62.

7. G. Simenon, Mon ami Maigret, Paris, Les Presses de la cité, 1952, p. 170.

8. A. Christie, The Big Four, Londres, Pan Books, 1927 , p. 24.

9. A. Christie, The Mirror Crack'd from Side to Side, Londres, Fontana, 1962, p. 152. 


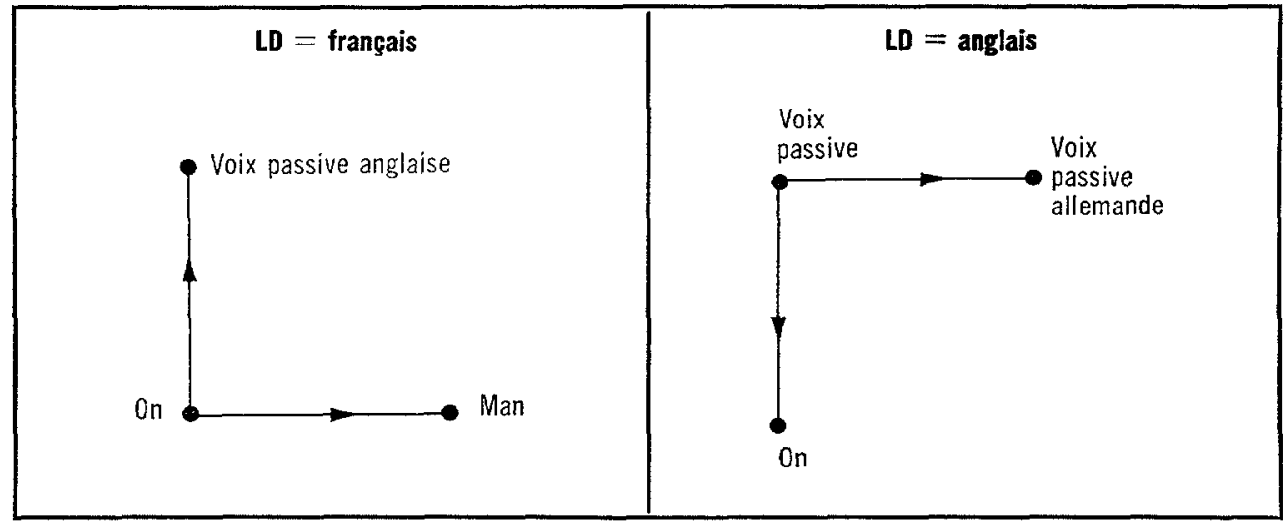

Et lorsque l'allemand devient LD, nous devrions pouvoir schématiser nos déductions hypothétiques de la façon suivante:

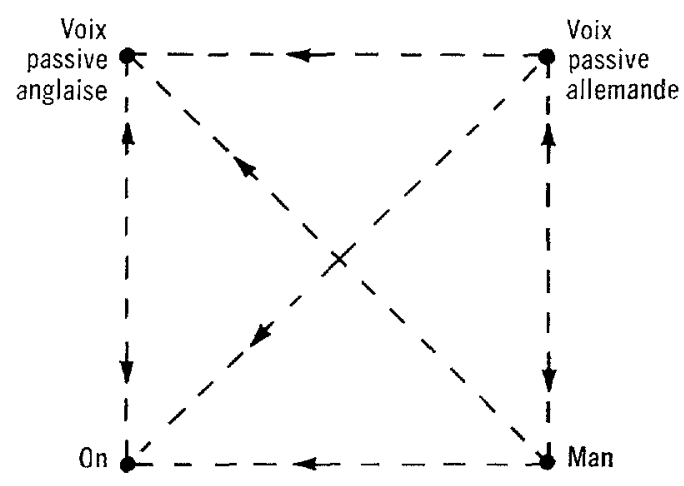

L'observation des exemples ci-dessous confirme nos hypothèses:

Er wird morgen begraben. ${ }^{10}$

On l'enterre demain.

He is to be buried to-morrow.

... sie war den Wölfen vorgeworfen worden ...11

... on l'avait jetée aux loups ...

... she had been thrown to the wolves ...

Seul le français reste constant; partout nous retrouvons la même valeur indéterminative du pronom « on ». Faut-il interpréter cette «fidélité » comme le fait $\mathrm{Ph}$. Lalanne lorsqu'il écrit:

Le français classique déteste l'impersonnel, au point de se créer des pronoms prétendument impersonnels qui lui donnent la satisfaction de mettre un sujet. L'anglais, comme le latin, comme bien d'autres langues, ne répugne point à laisser le sujet dans l'ombre et se plaît dans la voix passive. L'élégance des

10. G. Gaiser, Die sterbende Jagd, Francfort, Fischer, 1957, p. 70.

11. H. Böll, Billiard um halb zehn, Cologne, Kiepenheuer et Witsch, 1959, p. 126.

12. G. Gaiser, op. cit., p. 113.

Vol. $12-\mathrm{N}^{\circ} 2$ 
tournures françaises est fonction du sujet. L'anglais n'a cure d'élégance. Il la masque sous la brusquerie de formules brèves et impersonnelles. ${ }^{13}$

Le français serait donc l'élégance même, l'allemand n'aurait droit qu'à une demiélégance? Et que penser alors des affirmations d'A. Sauvageot qui écrit: "Cette construction n'est pas propre au français, puisqu'on la rencontre dans les langues telles que l'allemand, le suédois, le norvégien riksmaal et le danois par exemple. $\gg^{14}$ Nous sommes donc en droit de nous demander si le génie d'un peuple est dans la façon de s'exprimer ou dans le contenu de l'exprimé. Car, au fond, n'y a-t-il pas équivalence entre «on l'enterre demain » et «il sera enterré demain»? D'ailleurs, R. L. Wagner et J. Pinchon écrivent: «Contrairement à un préjugé tenace, l'emploi du passif est très vivant en français, aussi bien dans la langue parlée que dans la langue écrite. ${ }^{15}$

Nous pouvons donc reprendre notre schéma et remplacer les pointillés par des traits pleins:

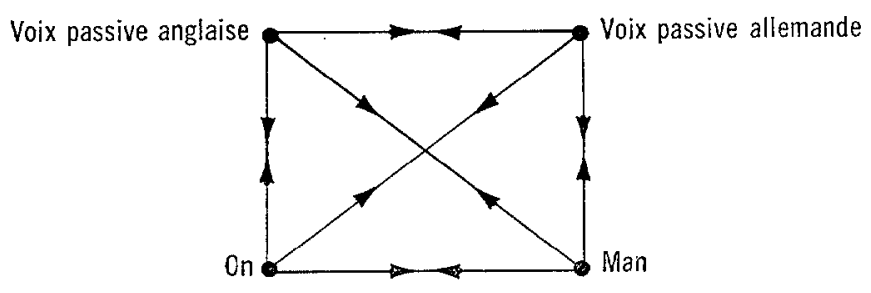

Nous pouvons aussi, pour être plus explicite, schématiser nos conclusions par un pentagone contenant une étoile à cinq branches. Chaque flèche symbolise une égalité soit licite, c'est-à-dire vérifiée par des exemples, soit déductive, c'est-à-dire obtenue par la juxtaposition raisonnée:

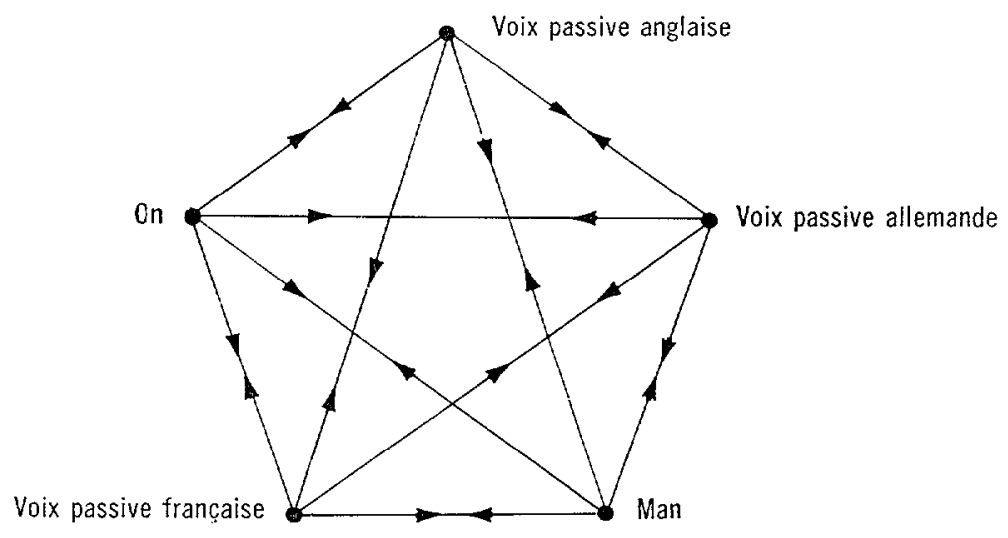

13. Ph. Lalanne, Mort ou renouveau de la langue française, Paris, André Bonne, 1957, p. 200.

13. Ph. Lalanne, Mort ou renouveau de la langue française, Paris, André Bonne,
14. A. Sauvageot, Français écrit, français parlé, Paris, Larousse, 1962, p. 132.

15. R. L. Wagner et J. Pinchon, Grammaire du français classique et moderne, Paris, Hachette, 1962, p. 283. 
Dans notre étude, ci-dessus, le contexte ne fournissait aucune indication permettant de substituer une valeur définie à l'indéfini. Nous pouvons alors établir la formule de la fonction primaire du pronom indéfini « on » de la façon suivante:

ON + INDICATION CONTEXTUELLE NÉGATIVE = FONCTION INDÉFINIE.

En généralisant cette équation, nous pouvons écrire:

FORME LINGUISTIQUE + INDICE = SIGNIFICATION.

Si la forme linguistique, dans nos exemples, demeure constante, l'indice variera cependant selon les données du contexte. Le nombre des variations contextuelles sera en rapport direct avec les multiples significations. Le contexte peut donc limiter d'une façon prégnante la valeur indéfinie « on » et le pronom peut admettre comme valeur d'arrière-plan celle de tous les pronoms personnels.

Les traducteurs ne doivent donc pas hésiter dans la substitution et accorder au pronom indéfini sa valeur définie, sa fonction secondaire.

\section{Exemples:}

On peut entrer? 18

Can $I$ come in ?

Darf man eintreten?

$I$ found it very interesting.$^{17}$

On y trouve des choses intéressantes ...

lch fand es sehr interessant ...

... und mit Gebäuden, auch wenn ich sie selbst erbaute, kann ich keine Versöhnung feiern. ${ }^{18}$ ... et, les aurait-on construits soi-même, on ne peut guère se réconcilier avec des édifices.

... and $I$ can't celebrate my reconciliation to a building, even if $I$ did built it myself.

... car on ne sait jamais ce que les gens penseront. ${ }^{19}$

... for you never know what people will think.

... denn man weiss nie, was die Leute sich bei so etwas denken.

You can't keep any secrets in St. Mary Mead. ${ }^{20}$

... on ne peut garder aucun secret ...

... kannst $d u$ nichts geheimhalten ...

... mit Brüder kannst $d u$ getrost einmal lachen ...21

... avec un frère on peut bien se permettre de rire ...

... amongst brother you can sometimes laugh ...

... les phrases qu'on vous répétera ...22

... the words she will repeat to you ...

... die Sä:ze, die Ihnen wiederholt werden ...

... kann er nicht, kauft er ihn ...23

... si on n'arrive pas à l'écraser, on l'achète ...

... if you can't do him down, buying him off ...

On ne s'est fâchés ni l'un ni l'autre.24

$W e$ 've already had things out between us.

Wir haben uns gegenseitig keinen Ärger gemacht.

$W e^{\prime}$ ll have these mecosoupies there ... ${ }^{25}$

On mettra ces pois de senteur là ...

Wir wollen den Mohn hierher pflanzen ...

... als wir im Frieden hier Blumen zogen. 26

... lorsqu'on y cultivait des fleurs.

... when we grew flowers.

C'est vrai ce qu'on raconte ? ${ }^{27}$

Is it true what they're saying ?

Stimmt es, was man erzählt ?

But what should they want to do that for ?28 Dans quel but aurait-on fait cela?

Aber zu welchem Zweck sollte man das getan haben?

16. G. Simenon, la Vérité sur Bébé Donge, Paris, Gallimard, 1942, p. 41.

17. A. Christie, The Mirror Crack'd from Side to Side, p. 166.

18. H. Böll, op. cit., p. 192

19. G. Simenon, Mon ami Maigret, p. 92.

20. A. Christie, The Mirror Crack'd from Side to Side, p. 24.

21. H. Böll, op. cit., p. 105.

22. G. Simenon, la Vérité sur Bébé Donge, p. 110.

23. G. Gaiser, op. cit., p. 117.

24. G. Simenon, Mon ami Maigret, p. 44

25. A. Christie, The Big Four, p. 7

26. G. Gaiser, op. cit., p. 120.

27. G. Simenon, les Vacances de Maigret, p. 153 .

28. A. Christie, The Big Four, p. 18. 
Da und dort hatten sie vergessen, das Radio $\mid$... on avait oublié de fermer la radio. abzustellen ...29 ... a radio had been left on.

Dans tous ces exemples, le contexte portait un indice positif. Nous pouvons donc écrire:

ON + INDICATION CONTEXTUELLE POSITIVE = FONCTION DÉFINIE.

L'on pourra peut-être objecter ici que les pronoms « you », «we », «they », « du », « sie » peuvent, eux aussi, consacrer une valeur indéfinie et qu'il y a identité parfaite entre ces pronoms et le pronom « on ». Mais, si « on» atteint d'emblée cette valeur, ces pronoms ne la rejoignent que par ricochet et ne sont que des «indéfinis » au deuxième degré où reste attachée leur valeur première de pronoms personnels.

Nous pourrions peut-être conclure avec W. Von Wartburg lorsqu'il écrit: "Le charme et la beauté de la langue française consistent très souvent à savoir supprimer ce qui n'est pas indispensable. ${ }^{30}$ Le traducteur doit donc retenir dans sa démarche traduisante la signification de la forme linguistique, signification dégagée, comme nous l'avons dit, par la rencontre d'une forme linguistique augmentée d'un indice contextuel. Si nous définissons le signe linguistique comme étant l'équivalent de la forme linguistique augmentée de son indice, nous pouvons écrire:

SIGNE LINGUISTIQUE $=$ SIGNIFICATION.

Si l'on admet, d'autre part, l'identité entre unité de traduction et signification, notre équation devient:

SIGNE LINGUISTIQUE $=$ UNITÉ DE TRADUCTION.

Nous savons que nous n'avons pas épuisé le relevé des valeurs du pronom « on », mais nous n'avons voulu que schématiser une démarche méthodologique de la fonction endogène d'une forme linguistique et du même coup étudier la démarche traduisante.

André Clas

29. G. Gaiser, op. cit., p. 20

30. W. Von Wartburg, Evolution et structure de la langue française, $7^{\mathrm{e}}$ éd., Berne, Francke, 1965 , p. 262. 